\title{
On the importance of background subtraction in the analysis of coronal loops observed with TRACE (Research Note)
}

\author{
S. Terzo ${ }^{1}$ and F. Reale ${ }^{1,2}$ \\ 1 Dipartimento di Scienze Fisiche ed Astronomiche, Università degli studi di Palermo, via Archirafi 36, 90123, Palermo, Italy \\ e-mail: terzo@astropa.unipa.it \\ 2 INAF - Osservatorio Astronomico di Palermo, Piazza del Parlamento 1, 90134 Palermo, Italy \\ e-mail: reale@astropa.unipa.it
}

Received 14 October 2009 / Accepted 5 February 2010

ABSTRACT

\begin{abstract}
Aims. Using TRACE coronal observations, we compare the analysis and diagnostics of coronal loop after subtracting the background with two different and independent methods.

Methods. We analyze sequences of images in the $171 \AA$ and $195 \AA$ filter bands of TRACE. One background subtraction method consists of considering background values obtained by interpolating between concentric strips around the analyzed loop. Another involves a pixel-to-pixel subtraction of the final image after the loop has completely faded out, used by Reale and Ciaravella.

Results. We compare the emission distributions along the loop obtained with the two methods and find that they differ considerably. We also find differences in the related filter ratio and temperature profiles. In particular, the pixel-to-pixel subtraction leads to coherent diagnostics of a cooling loop. After applying the other type of subtraction, the diagnostics are much less clear.

Conclusions. The background subtraction should be treated with care when analyzing a loop. The pixel-to-pixel subtraction appears to be more reliable, but its application is not always possible. Subtraction by means of interpolation between surrounding regions can produce higher systematic errors, because of intersecting structures and the large amount of subtracted emission in TRACE observations.
\end{abstract}

Key words. Sun: corona - Sun: X-rays, gamma rays - method: data analysis

\section{Introduction}

An important issue in the data analysis of coronal loop observations is background subtraction. Previous work (Del Zanna \& Mason 2003; Testa et al. 2002; Schmelz et al. 2003; Aschwanden \& Nightingale 2005; Reale \& Ciaravella 2006; Aschwanden et al. 2008) have established the importance of separating the true loop plasma from the diffuse foreground and background emission, produced by unresolved coronal structures and instrumental effects. Background subtraction is required because of the many overlapping bright structures and diffuse emission, nearby or along the line of sight, in addition to stray light (DeForest et al. 2009). The accurate extraction of emission along the loop is necessary before applying standard diagnostic methods (such as the filter ratio) to derive physical quantities, e.g., temperature, or applying more detailed loop models. When the background is significant relative to the loop intensity, it will seriously affect the extracted intensity along the loop. There is no standard and generally accepted method of background subtraction; the procedure is then "operator-sensitive" and so in turn are the results.

The problem is particularly important when analyzing observations where the background is a significant fraction of the signal. This happens, for instance, in observations performed with TRACE (e.g., Schmelz et al. 2003; Aschwanden \& Nightingale 2005; Reale \& Ciaravella 2006). Here we explore the dependence and sensitivity of the results on the background subtraction method, by comparing two methods applied to the same loop observed with TRACE in more than one filter band. We take advantage of the unique opportunity to apply two different and independent methods of background subtraction to the same dataset. One of the methods (Reale \& Ciaravella 2006, hereafter RC06) has the advantage that we use as background an image of the loop region when the loop is absent, and that background variations are estimated to be small throughout the observation.

In Sect. 2, we describe the data analysis and background subtraction. Section 3 shows and compares the results obtained by the two background subtraction methods, including implications for temperature diagnostics with filter ratios. In Sect. 4, we discuss the results.

\section{Data analysis}

The Transition Region and Coronal Explorer (TRACE) is a NASA Small Explorer (SMEX) mission to image the solar corona and transition region at high angular and temporal resolution, operating since 1998 (Handy et al. 1999). We analyze a TRACE observation of May 13 1998, with a 3.5h time sequence of $1024 \times 1024$ pixel full resolution image in two of TRACE filters ( $171 \AA, 195 \AA$ ). This is the same data set selected and analyzed by RC06. The same $512 \times 512$ pixel region of the whole field of view was extracted for the analysis. From these data, four images were selected, in each filter, at the times: 06:36:57 UT, 06:59:35 UT, 07:39:26 UT, and 08:29:34 UT for 171 A filter; and 06:37:18 UT, 07:00:06 UT, 07:39:47 UT, and 08:30:06 UT for $195 \AA$ filter. The filters have different sensitivities to the temperature of observed plasma. Observations taken at the same time with different filters provide information about the plasma 

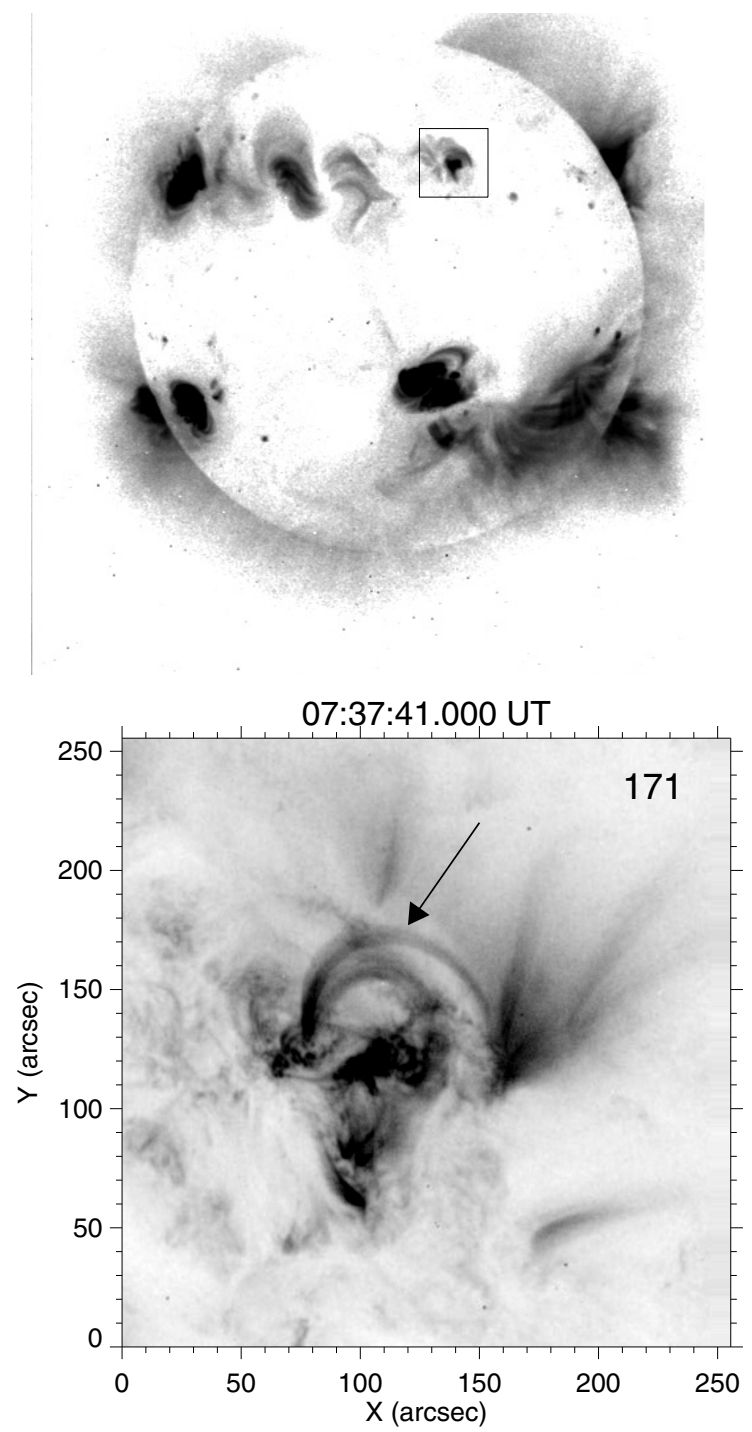

Fig. 1. Loop region as observed with TRACE $(256 \times 256$ pixels image $)$ in the $171 \AA$ filter at the labelled time (lower panel). The loop analyzed here is indicated by an arrow. The loop region is located in the inset of the Yohkoh/SXT full disk image (upper panel). The grey scale is inverted and linear for the TRACE image $(\leq 8 \mathrm{DN} / \mathrm{s} /$ pix $)$ and inverted and logarithmic for the Yohkoh image (between 10 and $150 \mathrm{DN} / \mathrm{s} / \mathrm{pix}$ ).

temperature. In particular, the ratio of the emission in two different filter bands, relative to the ratio of the response functions of the filters, infers the temperature of the emitting plasma. Since the filter ratio may be altered by the diffuse emission, originating both in other structures coaligned along the line of sight and in the stray light, it is very important to estimate and remove this background emission. In TRACE observations, the diffuse emission is typically very high and might represent most of the signal.

\subsection{Loop analysis}

We analyze the same loop as in RC06. The loop is selected on the TRACE images (Fig. 1), and appears as an entire loop in several $171 \AA$ filter images (Fig. 2). It is clearly visible in the $195 \AA$ filter band (Fig. 2). The loop is bright, i.e., observed with high quality statistics and a high contrast over the background. It was selected as far as possible to be free from other structures intersecting along the line of sight. The loop evolves in both filter bands. In the $171 \AA$ band, it is faint initially, then brightens reaching a peak of intensity around 07:30 UT, and it fades again, before disappearing at the end of the sequence ( 10:00 UT, RC06). In the $195 \AA$ band, the loop is initially brightest and progressively fades out. This loop evolution is even clearer in Fig. 3, which shows images of the loop region normalized to the final image at $10 \mathrm{UT}$, where the loop is no longer visible. Here we revisit the data at the four selected times. The data were treated with the standard procedures for TRACE data processing contained in the Solar SoftWare (ssw) and the images were coaligned using a standard cross-correlation technique.

\subsection{Background subtraction}

We compare the method previously adopted in RC06 with an alternative and independent approach. This method is based on interpolation between emission values in a region close to (but outside) the loop, and is similar to the method adopted in Testa et al. (2002), Aschwanden \& Nightingale (2005), Schmelz et al. (2003) and Aschwanden et al. (2008). As a first step, we measure the loop emission. We define a strip enclosing the loop in both of the TRACE filter passbands, and divide the strip into sectors, as shown in Fig. 4. We analyzed strips of different widths. A width of 10 pixels is a good compromise between too low quality statistics and exceeding too much the loop borders. With this choice, we obtain 27 similar almost square sectors (RC06). We then mark two other strips, parallel and concentric to the one used to extract the loop emission, one outside and another inside the imaginary circle of which the loop is part. We divide these two additional strips into sectors aligned with those of the central strip (Fig. 4). These two other strips are used to extract the emission for background subtraction. We place them as close as possible to the loop of interest, avoiding other nearby structures that differ from the analyzed loop. Both strips also have a width of 10 pixels and are divided into 27 sectors to ensure a one-toone correspondence of these sectors with the sectors patching the loop. In each of the 27 sectors in the two external strips, we compute the mean emission value per pixel. To each sector of the central strip, we assign a background equal to the emission linearly interpolated between the two corresponding sectors of the external strips (Fig. 4).

To ensure completeness, we also considered a least squares quadratic interpolation. We then considered two additional concentric strips about the analyzed loop, one outer and the other inner. However, the additional outer strips are so far from the central loop they include other bright structures that severely contaminate the procedure and locally alter the otherwise smooth emission distribution. Therefore, the higher order interpolation does not in our case improve our results. Nevertheless, we point out that we linearly interpolate between values obtained by averaging over a sector. This is already and intrinsically more accurate than a linear interpolation performed between single pixels.

We recall that the method of background subtraction of RC06 assumes that the loop disappears at the end of the image sequence. The last image (around $10 \mathrm{UT}$ ) is then subtracted pixel-by-pixel from all other images, where the structures surrounding and crossing the loop of interest along the line of sight do not change much during the observation sequence. To estimate the background fluctuations during the observation, RC06 measured an average pixel-by-pixel standard deviation of $13 \%$ in both the $171 \AA$ and the $195 \AA$ filter band. 

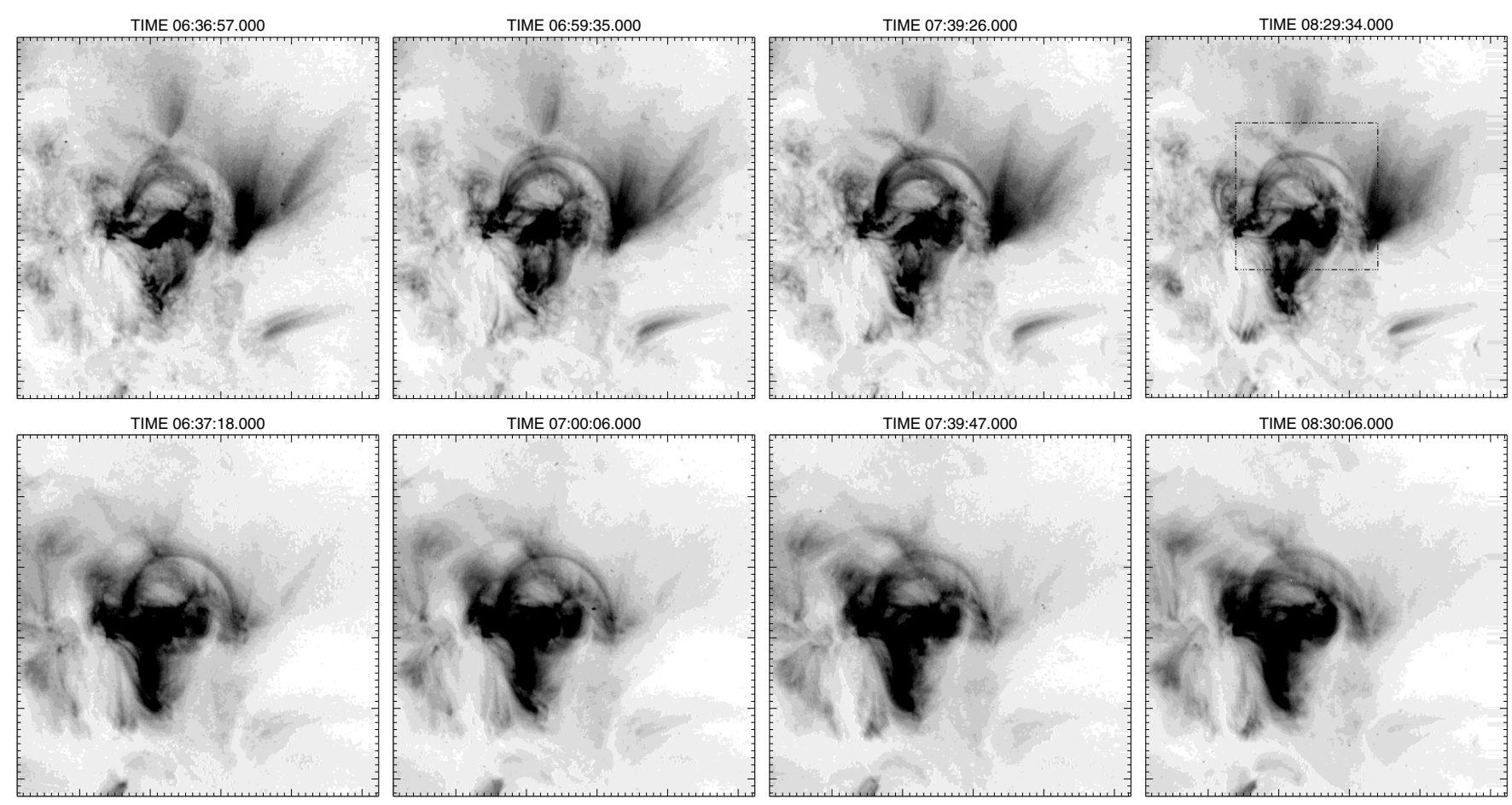

Fig. 2. Loop region (top row: $171 \AA$; bottom row: $195 \AA$ ) at subsequent times (from left to right): 06:36:57.000 UT, 06:59:35.000 UT, 07:39:26.000 UT, 08:29:34.000 UT for 171 $\AA$, and 06:37:18.000 UT, 07:00:06.000 UT, 07:39:47.000 UT, 08:30:06.000 UT for 195 $\AA$. The grey scale is inverted and linear between 0.4 and $7 \mathrm{DN} \mathrm{s}^{-1} \mathrm{pix}^{-1}$ for all images. The frame in the top right image is the part shown in Fig. 3 .

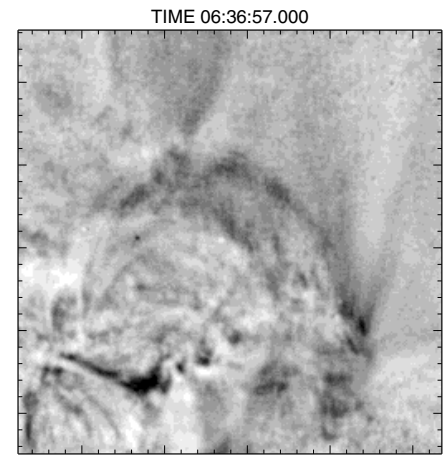

TIME 06:37:18.000

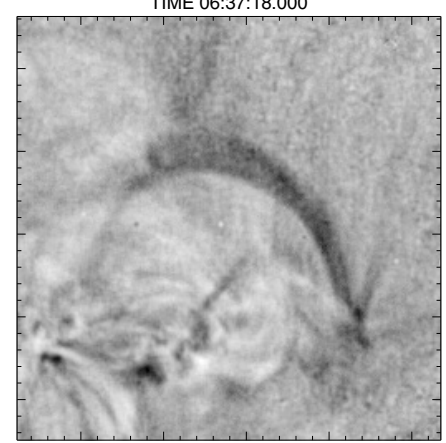

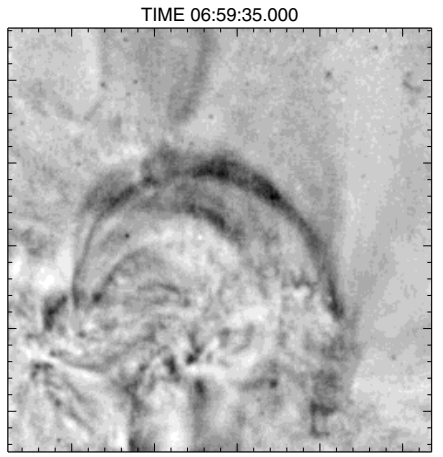

TIME 07:00:06.000

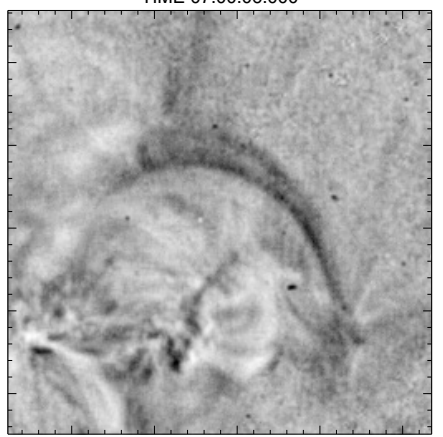

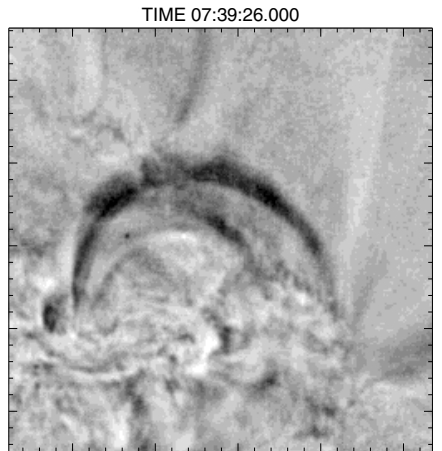

TIME 07:39:47.000

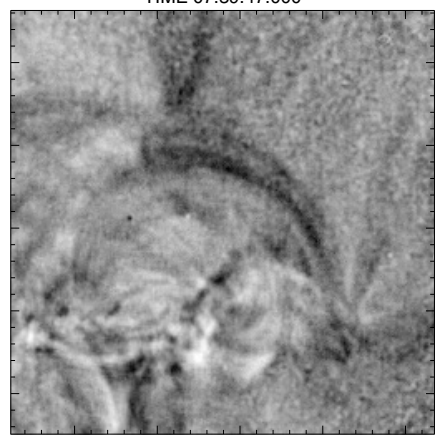

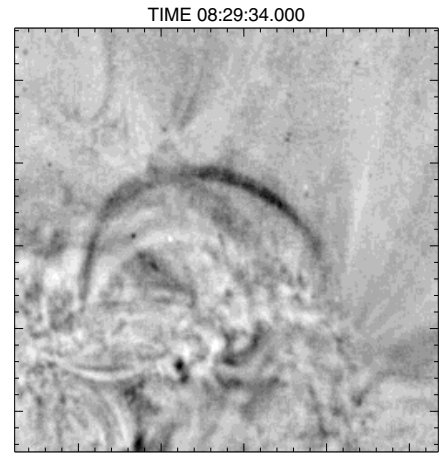

TIME 08:30:06.000

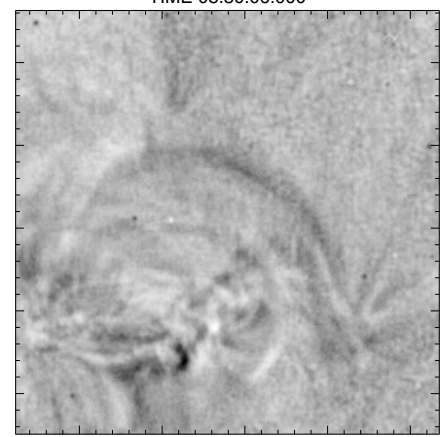

Fig. 3. Zooms of the loop region in Fig. 2 (frame in the top right image of Fig. 2). The images (top row: $171 \AA$; bottom row: $195 \AA$ ) are normalized to the final image of the sequence (at 10:00 UT), where the loop is no longer visible, to enhance the contrast of the loop evolution. The grey scale is linear between 0.4 and 2.5 ( 1 means that the emission value is the same as that of the final image).

A more accurate subtraction would be achieved if the subtracted image were produced by interpolating between an image before the loop switches on and one after the loop switches off. Unfortunately, this loop has a very long lifetime and to find images acquired before the loop ignition we search more than $25 \mathrm{ks}$ earlier than the start time of our analysis. In our opinion, this time is too long for us to assume that the environment did not change and to measure a reliable background, and we prefer to keep the final image as the only image for subtraction.

We also point out that the image to subtract was taken at 10:00 UT, significantly apart in time from the analyzed images taken between 06:30 and 08:30 UT. The perspective (i.e., viewing angle) of the observed loop varied during that interval possibly impacting the RC06 scheme, which assumes that each line of 

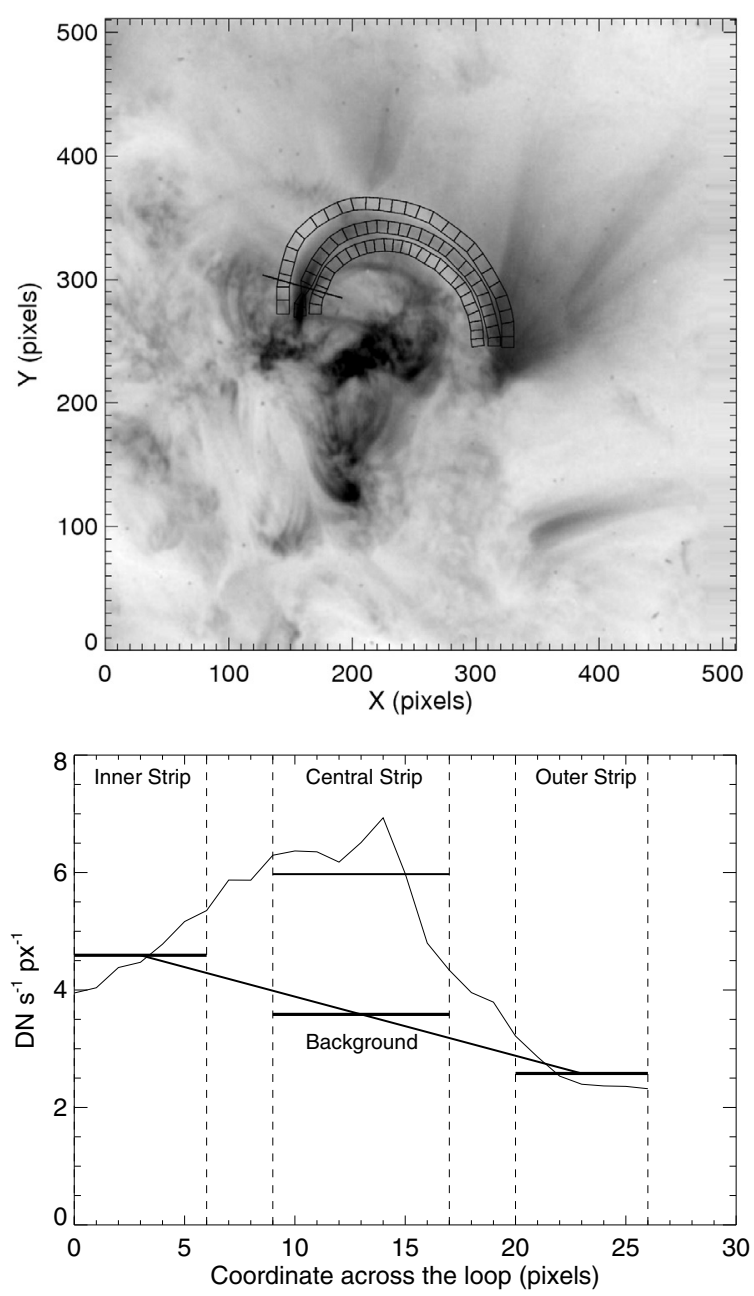

Fig. 4. Background subtraction with interpolation method. Upper panel: the strips for the loop analysis (central) and for the background subtraction (outer and inner) are marked in the loop region (07:39:26.000 UT, $171 \AA$ filter). Lower panel: Emission profile (thin solid line) measured for a cut across the loop (marked in the upper panel). We compute the average emission (thick horizontal lines) in the corresponding sectors intersected by the outer and inner strips (bounded by vertical dashed lines). The background value (central horizontal line) is computed by linear interpolation of the average emission values of the outer strips. This is then subtracted from the total emission measured in the central strip (upper solid line).

sight intersects the same column of plasma throughout the entire sequence. However, we do not expect a significant effect since we estimate a change in the viewing angle of the order of $1-2^{\circ}$ or less, as confirmed by a visual inspection of the images after subtraction.

These are not the only two methods of background subtraction. Schmelz et al. (2007) and Schmelz et al. (2003) used two more approximate methods of background subtraction: (1) constant background subtraction; and (2) pixel pair background subtraction. In the former method, they subtract a constant value extracted from a single background pixel chosen above the loop apex; in the latter, they selected a background pixel for each loop pixel.

\section{Results}

\subsection{Loop emission}

Figures 5 and 6 show examples of applying our interpolation method of background subtraction. The lines join the data points, which are the mean values of the pixel emission in a sector. The error in each mean value is the standard deviation of the mean in that sector. The error was conservatively rounded to 0.05 . The lower panels of Figs. 5 and 6 show the results subtracting values obtained by interpolating between the dotted and the dashed curves, from the solid curve.

The loop emission is diminished significantly by the background subtraction, at some points even completely cancelled, because of the overlap with other bright structures close to but independent of our loop. To directly comparison the interpolation and the RC06 background subtraction methods, we plot the emission along the loop obtained with the two methods in the same figure. Figure 7 shows the results for both the $171 \AA$ filter and the $195 \AA$ filter. We can see that the emission significantly differs between the two methods: the profiles mostly differ in the central part, which is predicted to be very faint at any time after background subtraction using the interpolation method. In contrast, with the latter method the footpoints are found to be mostly brighter than with the pixel-to-pixel subtraction, although we observe agreement at times (e.g., at the right extreme at 06:36 UT and 07:00 UT in both filters). The footpoint result is debatable, since the footpoint regions cannot be clearly resolved in the original data (Fig. 2). There is no doubt, however, that the bulk of the loop is clearly visible at many times (Figs. 2 and 3), and therefore, the emission cannot be so low, in contrast to the interpolation method and in agreement with pixel-to-pixel method. Therefore, when the two methods differ the most, RC06's pixelto-pixel method surely provides a more reliable result. There is some agreement between the profiles at the latest reported time.

We note in Fig. 7 that, since the emission remaining after subtraction by the interpolation method is faint except close to the loop footpoints, it does not clearly evolve with time. RC06 found that the background-subtracted central section of the loop evolves in agreement with the evolution observed in the images (Figs. 2 and 3). Therefore, the results obtained with the RC06 method appear more reliable than those obtained with the interpolation method.

\subsection{Temperature diagnostics}

To establish the differences between the background subtraction methods, we analyzed the temperature diagnostics obtained from the filter ratio. It is well-known that for an optically thin plasma, which is isothermal along the line of sight, the ratio of the emission detected in two different filters is a function of the temperature only (e.g., Rosner et al. 1978). From the ratio value measured in an image pixel we can then derive a temperature value in that pixel and compile a proper thermal map. For the narrow-band $171 \AA$ and $195 \AA$ filters of TRACE, the relationship between temperature and filter ratio $195 / 171$ is monotonic only across a certain temperature range, i.e., $0.7<T<1.8 \mathrm{MK}$ (e.g., Aschwanden et al. 2000), the range for which the filter responses are the highest. It is reasonable to assume that the detected plasma is all in that temperature range and this allows us to measure the temperature along the loop, after background subtraction. As an example, Fig. 8 shows the filter ratio 195/171 and the corresponding temperature along the loop at 07:40 UT computed after the background subtraction using the pixel-to-pixel 

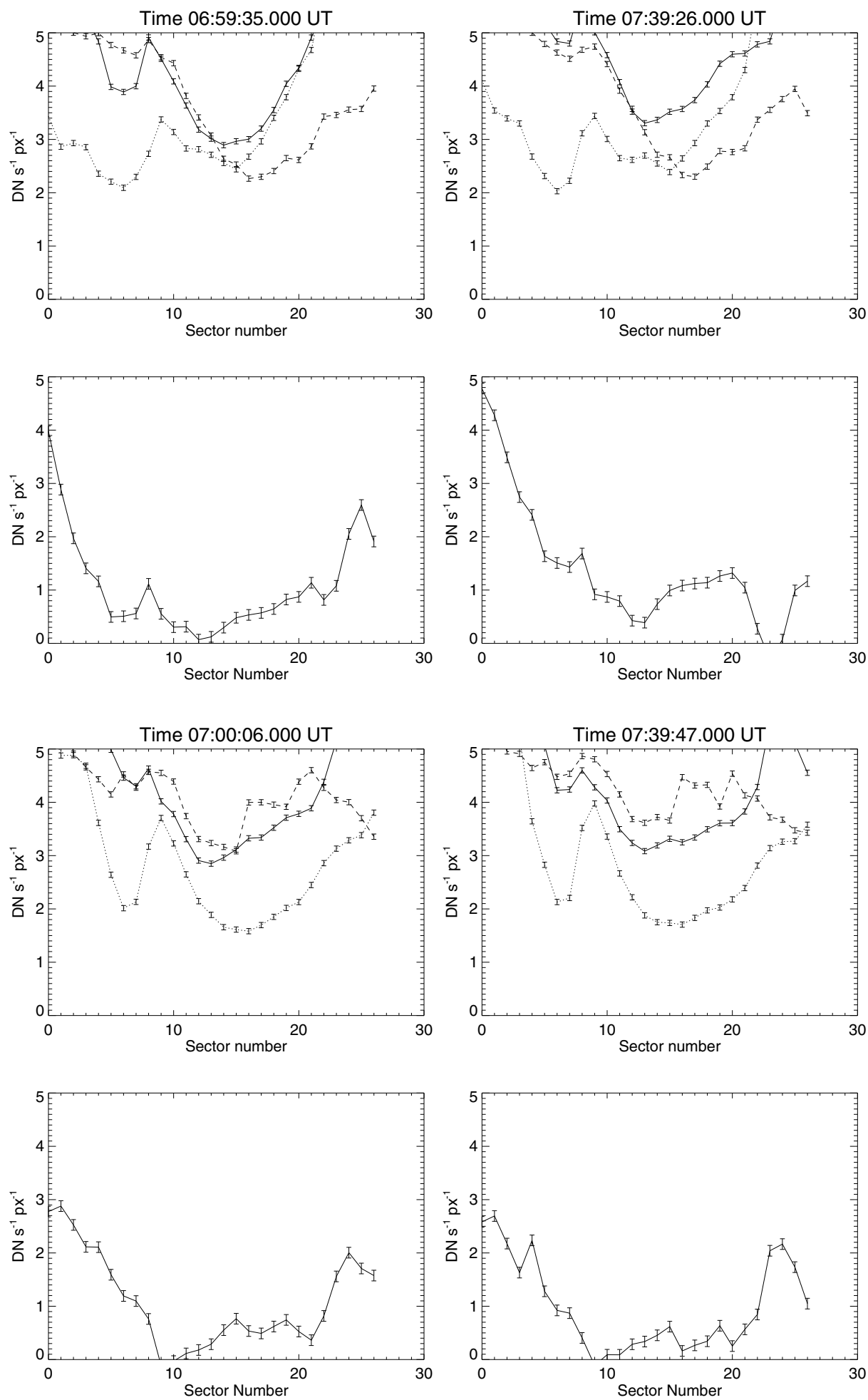

Fig. 5. Emission along the loop in the $171 \AA$ filter, at 06:59:35.000 UT (left column), and 07:39:26.000 UT (right column). Upper panels: emission along the loop strip (solid line), outer strip (dotted line), and inner strip (dashed line). Lower panels: emission after background subtraction with the interpolation method.

Fig. 6. As Fig. 5 for the $195 \AA$ filter, at 07:00:06.000 UT (left column), and 07:39:47.000 UT (right column). method (also shown in RC06) and the interpolation method. The ratio profile obtained with the latter method appears to be far more irregular, so we are unable to define an overall trend. In contrast, the other method produces a more regular profile, which is coherent with the clear visibility of the loop in the filter ratio map (RC06), despite the dip at the left leg caused by another crossing bright structure. We find similar trends in the corresponding temperature profiles along the loop.

We emphasize that, although both profiles indicate overall a small temperature variation along the loop, the quality of the pixel-to-pixel background determines whether a trend can be identified or not. The globally flat temperature profile is typical of TRACE data (e.g., Lenz et al. 1999) and was attributed in part to an instrumental bias (Weber et al. 2005). However, RC06 showed that the filter ratio diagnostics are anyhow meaningful and sensitive enough to detect the loop progressive cooling. Figure 9 shows the loop average filter ratio obtained after the two different background-subtraction methods at four different times. From the evolution obtained with the interpolation method, it is far less clear that the loop is cooling, confirming that the sensitivity of the temperature diagnostics is higher with the pixel-to-pixel background subtraction. 

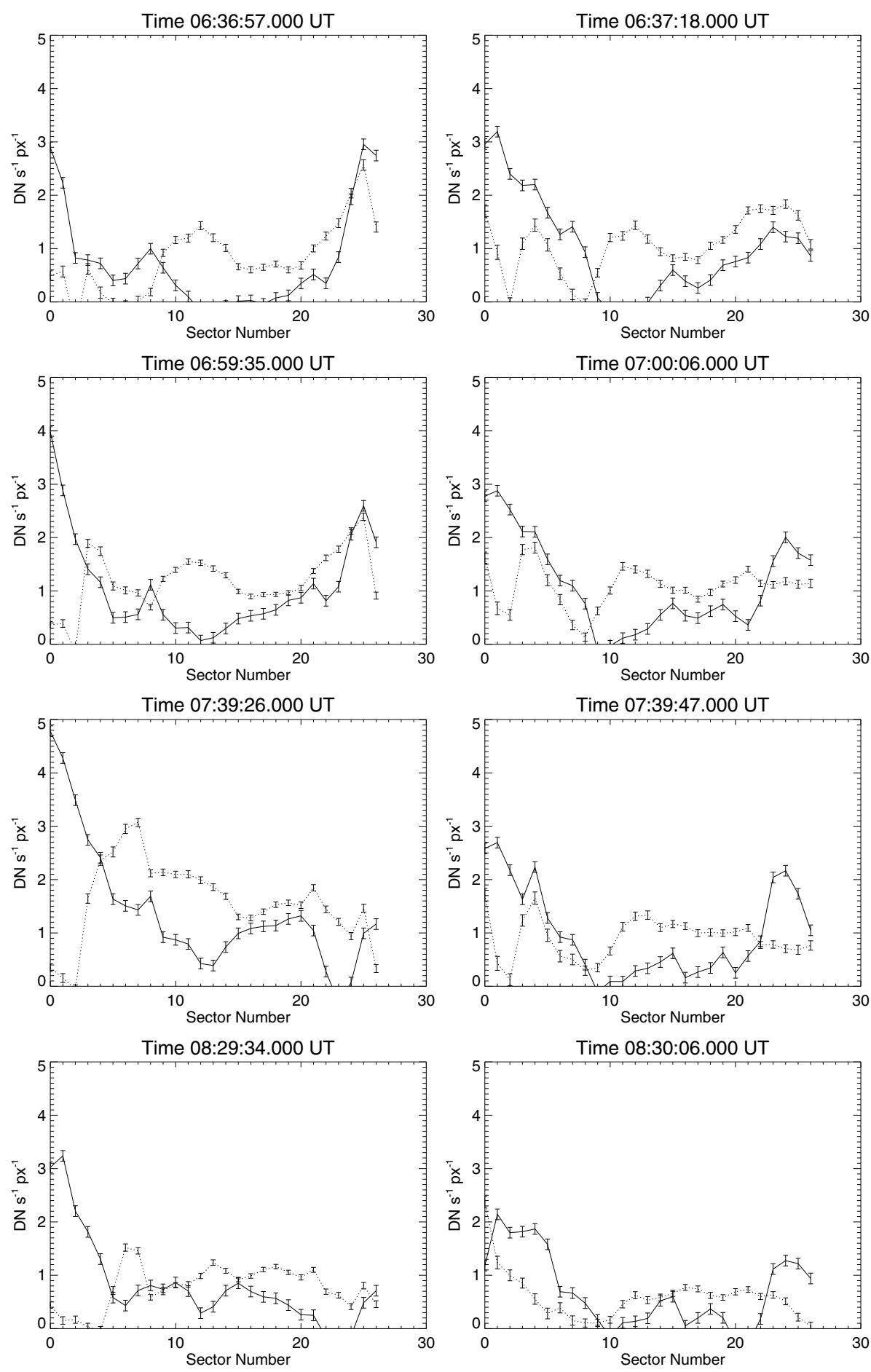

Fig. 7. Emission along the loop at the labelled times in the $171 \AA$ filter (left column) and the $195 \AA$ filter (right column). All panels show the emission along the loop, after background subtraction with two different methods: pixel-bypixel method (RC06, dashed line), and interpolation method (solid line).

\section{Discussion}

We have demonstrated that the accuracy of the background subtraction can significantly affect the quality of the analysis of TRACE coronal loops because the background signal is very high and influenced by many bright structures near and perhaps entangled with the analyzed loop, along the line of sight. We have taken advantage of a unique opportunity to compare two different and independent methods of background subtraction: one that subtracts the interpolated emission of two off-loop strips; and the other that subtracts pixel-by-pixel the complete image after the disappearance of the loop (RC06). Extracting the background emission with two strips as close as possible to the loop of interest, we have been able to remove emission from structures that can transversally intersect the loop. This is true as long as the "contaminating" loops run across the target loop, while it is not true when this occurs at an oblique angle.

We have shown that not only do different methods produce different emission profiles, but that the subsequent diagnostics are affected. With the pixel-by-pixel subtraction, we are able to derive coherent filters ratio profiles along the loop and measure a coherent temperature evolution where the loop globally cools as expected from the sequence of appearance/disappearance in the different TRACE filters. These results are far less clear after using the other method of background subtraction. 

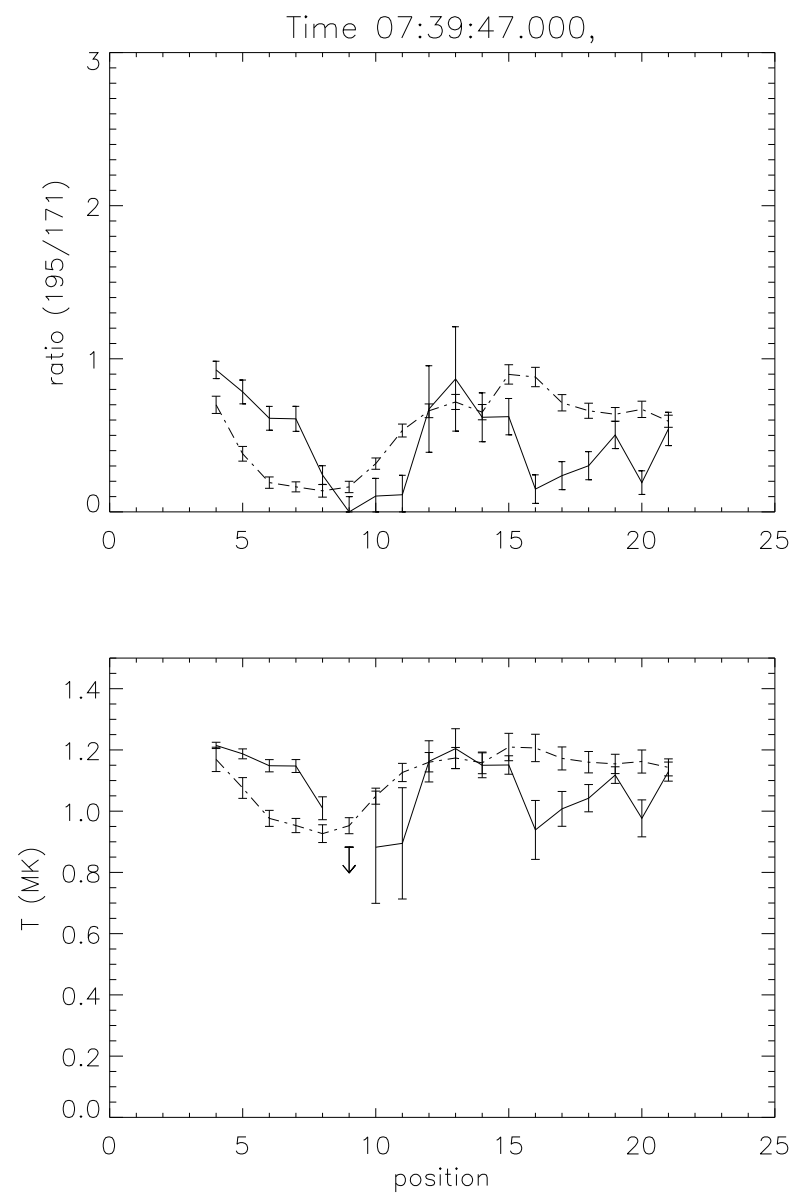

Fig. 8. Filter ratio 195/171 (top) and corresponding temperature (bottom) along the loop at 07:40 UT computed after background subtractions with either the pixel-to-pixel (dashed) or interpolation (solid) methods. The arrow is an upper limit to the interpolation data.

The RC06 background subtraction therefore looks to be a more reliable method. In principle, it is accurate, if the loop of interest is the most variable structure in the field of observation; it is direct, in contrast to the interpolation method; it is applied pixel-by-pixel, allowing us to derive "background-subtracted images" and therefore to achieve a visual feedback by analyzing all loop pixels, instead of sampling them at selected positions. On the other hand, this method works well as long as the structures surrounding and crossing the loop of interest along the line of sight do not change much during the observation. In other words, the RC06 method cannot take time variations in the background emission into account and we cannot exclude that crossing structures vary during the observation. We also remark that the pixel-by-pixel subtraction could be applied only because the loop disappears at the end of the image sequence. This condition can be matched only by evolving loops. For loops that remain steady during the entire observation, other methods must be used. The interpolation of out-loop emission has often been used (Testa et al. 2002; Aschwanden \& Nightingale 2005; Schmelz et al. 2003; and Aschwanden et al. 2008), but according to our analysis, it may lead to severe systematic errors when affected by other structures close to but distinct from the loop under analysis.

Figure 4 illustrates the difficulties encountered by the interpolation method by showing that close to the target loop there is another structure of similar intensity. Although the interpolation method should reliably estimate the background around the two

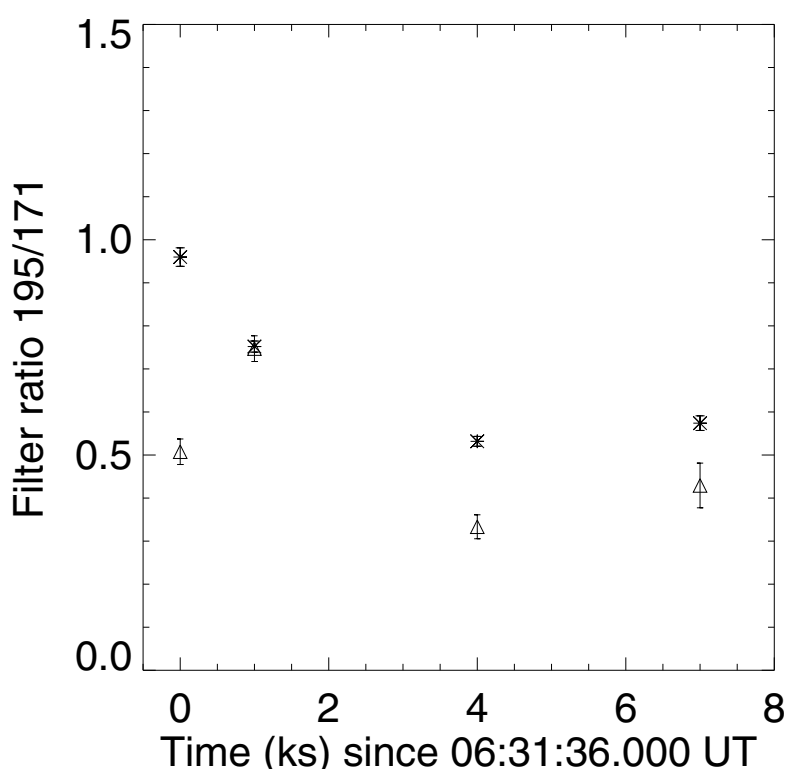

Fig. 9. Evolution of the loop average 195/171 filter ratio, sampled at the four times analyzed in this work and obtained by applying background subtractions, with either the pixel-to-pixel (stars) or interpolation (triangles) methods.

loops, it is unable to take the overlap of the two loops properly into account. A scheme that fits separately the two loops and their respective backgrounds estimated as above may provide a more accurate solution.

Other methods of background subtraction are instead too "operator-sensitive", because they are based on the meticulous selection of single background pixels, intentionally avoiding in this way structures that can cross the loop of interest and significantly alter the loop emission.

In conclusion, this work confirms and qualifies how a reliable background subtraction is a delicate and difficult task when analyzing coronal loops observed with TRACE. The problem can of course be greatly reduced by performing observations with instruments that have far lower instrumental background emission and hence lower systematic errors.

Acknowledgements. We thank the anonymous referee for constructive suggestions. We acknowledge support from Italian Ministero dell'Università e Ricerca and Agenzia Spaziale Italiana (ASI), contract I/023/09/0.

\section{References}

Aschwanden, M. J., \& Nightingale, R. W. 2005, ApJ, 633, 499

Aschwanden, M. J., Tarbell, T. D., Nightingale, R. W., et al. 2000, ApJ, 535, 1047

Aschwanden, M. J., Nitta, N. V., Wuelser, J.-P., \& Lemen, J. R. 2008, ApJ, 680, 1477

DeForest, C. E., Martens, P. C. H., \& Wills-Davey, M. J. 2009, ApJ, 690, 1264 Del Zanna, G., \& Mason, H. E. 2003, A\&A, 406, 1089

Handy, B. N., Acton, L. W., Kankelborg, C. C., et al. 1999, Sol. Phys., 187, 229

Lenz, D. D., Deluca, E. E., Golub, L., et al. 1999, Sol. Phys., 190, 131

Reale, F., \& Ciaravella, A. 2006, A\&A, 449, 1177

Rosner, R., Tucker, W. H., \& Vaiana, G. S. 1978, ApJ, 220, 643

Schmelz, J. T., Beene, J. E., Nasraoui, K., et al. 2003, ApJ, 599, 604

Schmelz, J. T., Roames, J. K., \& Nasraoui, K. 2007, Adv. Space Res., 39, 1497

Testa, P., Peres, G., Reale, F., \& Orlando, S. 2002, ApJ, 580, 1159

Weber, M. A., Schmelz, J. T., DeLuca, E. E., \& Roames, J. K. 2005, ApJ, 635, L101 